\title{
Un viaje por el tiempo
}

\author{
José Rubén Morones Ibarra
}

Universidad Autónoma de Nuevo León, Facultad de Físico-Matématicas

rubenmorones@yahoo.com.mx

\section{RESUMEN}

En este artículo se analiza la evolución que ha tenido el concepto tiempo en la ciencia. El tiempo como un concepto elemental que se forma en nuestra mente simplemente como resultado de nuestra existencia, y que nos permite ordenar los acontecimientos, ha resultado ser uno de los más misteriosos y difíciles de comprender. El tiempo lo introdujo Newton en la ciencia como un parámetro absoluto que no resulta afectado por nada, usándolo como un recurso matemático para describir los fenómenos de la naturaleza. Posteriormente, Einstein encontraría que el tiempo no es una cantidad absoluta, sino que depende del observador que lo mida. Actualmente se piensa que el tiempo, que parece que fluye como un continuo, puede ser discreto. Además, es posible que el pasado, que creemos que es algo que dejó de existir, puede ser revisitado con la posibilidad de los viajes en el tiempo.

\section{PALABRAS CLAVE}

El concepto tiempo, el tiempo en la ciencia, el tiempo en la relatividad, cuantizacion del tiempo, viajes en el tiempo.

\section{ABSTRACT}

In this article the evolution of the concept of time in science is analyzes. Time as an elementary concept that is formed in our mind simply because of our existence, and that allows us to order events, has turned out to be one of the most mysterious and difficult to understand. Time was introduced by Newton in science as an absolute parameter that is not affected by anything, using it as a mathematical resource to describe the phenomena of nature. Later, Einstein would find that time is not an absolute quantity but depends on the observer that measure it. Time, which seems to flow as a continuum, could be discrete. Furthermore, it is possible that the past, which we believe to be something that ceased to exist, can be revisited with the possibility of time travel.

\section{KEYWORDS}

Concept of time, time in science, time in relativity, time cuantization, timetravels.

\section{INTRODUCCIÓN}

Los tres conceptos fundamentales y más primitivos que aparecen en nuestra mente en forma natural, como resultado solamente de nuestra existencia y capacidad de observación, son los de espacio, tiempo y materia. Podríamos agregar, muchos más, como el de movimiento, por ejemplo, pero este concepto queda relacionado finalmente con lo que se mueve, que es materia, y con el espacio y el tiempo. Así que el movimiento puede ser descrito en términos de los 
conceptos más fundamentales ya mencionados. Podemos concluir que espacio, tiempo y materia son más básicos que el concepto de movimiento. Movimiento es algo que le ocurre a la materia, o es una forma en la que se presenta la materia.

En este artículo se abordarán algunas ideas asociadas con el concepto tiempo. Encontraremos que este concepto no es tan simple como puede parecer desde el punto de vista de nuestra intuición. Por otra parte, algunos temas tratados aquí, como el de la construcción de una teoría física donde no aparezca el tiempo o el asunto de los viajes en el tiempo, no son tópicos que pertenezcan a la ciencia de ficción o a ideas fantásticas, por el contrario, todo ello está enmarcado estrictamente dentro de la ciencia moderna y es actualmente objeto de estudio.

El famoso cosmólogo Stephen Hawking solía mencionar que se pueden establecer tres conceptos asociados con el tiempo. El primero de ellos es el de "tiempo psicológico". Este concepto corresponde a nuestra percepción del tiempo como seres humanos. En él distinguimos el pasado, el presente y el futuro. El pasado lo identificamos mediante el recuerdo. Recordamos el pasado, pero no cosas del futuro. El presente es lo que estamos viviendo, y el futuro es algo desconocido. Además, sabemos que nos movemos hacia adelante en el tiempo, es decir, hacia el futuro, porque el número de cosas que podemos recordar está aumentando.

Una segunda idea del tiempo es el del "tiempo entrópico", el cual está asociado con el concepto termodinámico de entropía. Las ideas detrás del concepto de tiempo entrópico están relacionadas con la observación de que los fenómenos naturales ocurren de tal manera que el desorden total del universo aumenta siempre. Como ejemplo citamos el hecho de que, si ponemos una gota de tinta en un vaso con agua, la tinta empieza a difundirse en el agua. Ese es el proceso natural. Esto define la flecha del tiempo, como evolucionando del orden hacia el desorden. No esperamos que ocurra lo contrario, que una vez que la tinta se ha diluido en el agua, ocurra el proceso inverso de que todas las pequeñas piezas de tinta diluidas en el agua inicien un proceso inverso de aglomeración y que formen una gota como la que se colocó inicialmente.

El tercer concepto de tiempo es el concepto de "Tiempo cosmológico". Este está asociado con la evolución del universo desde la Gran Explosión hacia el crecimiento o inflación del universo. El tiempo cosmológico $t$, corresponde a la edad del universo. Se establece que el tiempo inició con la Gran Explosión. Cuando empiece el periodo de contracción del universo, si es que esto ocurrirá, se invertirá la flecha o dirección del tiempo.

El gran problema con el tiempo cosmológico es que no se puede establecer el inicio del tiempo desde un formalismo científico, asociándole un fenómeno físico, que fue el momento del Gran Estallido, ya que esto corresponde a una singularidad cosmológica en la Teoría General de la Relatividad (TGR). La TGR ha sido utilizada en la cosmología para describir le evolución del Universo como un todo, sin embargo, esto no está exento de singularidades. Para evitar singularidades de esta naturaleza, Hawking propuso un tratamiento de tiempo imaginario para analizar el origen y evolución del universo. No ha quedado resuelto aún este problema.

\section{LA NATURALEZA DEL TIEMPO}

El concepto tiempo es sin duda uno de los más abstractos, complejos y misteriosos en la vida del ser humano. La pregunta ¿qué es el tiempo?, tiene una profunda dificultad para ser contestada. Cualquier respuesta que demos a ella es susceptible de ser cuestionada y puesta en duda. Sin embargo, existe el tiempo de nuestra experiencia, el tiempo empírico que da forma al concepto de tiempo intuitivo. Después de la teoría de la relatividad el tiempo absoluto en el que creíamos se derrumbó para dar paso a la relatividad del tiempo. Después vendría la teoría cuántica que dio origen a nuevas ideas sobre el tiempo. 
Mostraremos, con algunos ejemplos sencillos que lo que consideramos intuitivamente como tiempo es bastante engañoso, y que los conceptos intuitivos que tenemos del tiempo son en realidad equivocados. La percepción humana del tiempo entra en conflicto con el concepto relativista del tiempo. Sin embargo no es necesario adentrarnos en el estudio de la teoría de la relatividad para encontrar algunas dificultades entre nuestra idea de tiempo y lo que la ciencia nos enseña. Con solo admitir el valor finito y constante de la velocidad de la luz, podemos ir entendiendo y aceptando esa diferencia entre el sentido común y el razonamiento científico cuando tratamos al tiempo.

Mientras no nos hagamos la pregunta de ¿qué es el tiempo?, todo parece muy claro sobre el tiempo. Pero en el momento en que tratemos de responder a la pregunta nos encontramos con serias dificultades. Esto se deba a que el tiempo intuitivo debe cumplir con ciertas propiedades, y esto lo convierte en un concepto no simple.

Ilustremos las dificultades que se presentan detrás de las ideas y expresiones sobre el tiempo. El tiempo lo asociamos con eventos que se suceden uno después de otro. El presente es por supuesto diferente del pasado y también lo será del futuro. Sin embargo lo que observamos actualmente pertenece en realidad al pasado. Al ver a una persona que se encuentra a 5 metros de distancia, por ejemplo, lo estamos viendo, al captar su imagen que nos trae la luz, como era hace algunos nanosegundos. Al ver a la Luna lo que captamos es su posición y estado hace un segundo, no en el presente. Al observar una estrella lo que vemos es cómo era hace varios años o millones de años, dependiendo de la distancia a la que se encuentre.

El concepto de "en este momento" o "ahora" es un concepto local, que depende de la capacidad de nuestros relojes para establecer diferencias entre intervalos de tiempo. En el Sol, lo que ocurre en este momento, lo podemos saber solo 8 minutos después, no en este instante. Así que el "ahora" no tiene en general significado físico, solo lo tiene localmente.

\section{EL TIEMPO EN LA CIENCIA}

El concepto tiempo en la ciencia ha sufrido modificaciones durante la evolución del conocimiento científico. En la teoría Newtoniana de la mecánica el tiempo fluye sin ninguna relación con nada, con ningún fenómeno. En las teorías físicas anteriores a Einstein el tiempo es un parámetro invariante sin conexión con nada. El tiempo lo incluyó Newton en su teoría de la mecánica como un recurso matemático para la descripción de los fenómenos naturales.

Con la teoría de la relatividad de Einstein, el tiempo se convierte en un actor en la escena de los procesos físicos, no como parte del escenario. El tiempo, el espacio y la materia están estrechamente conectados, no existe el uno sin el otro. El tiempo se deforma, tiene arrugas, es algo elástico. La estructura del espacio-tiempo resulta ser un campo dinámico, como el campo electromagnético o el campo de otras fuerzas de la naturaleza.

\section{LA CUANTIZACIÓN DEL TIEMPO}

En las teorías cuánticas, muchas cantidades físicas están cuantizadas. Esto significa que no tienen valores continuos sino discretos. La energía, por ejemplo, aparece en paquetes llamados cuantos de energía, lo mismo que la luz, aparece en cuantos llamados fotones. Cuantizar es equivalente a granular y tener solo granos de cada cantidad.

En la teoría general de la relatividad, la gravitación es una manifestación de la curvatura del espacio-tiempo. En las ondas gravitacionales que se descubrieron en el año 2017, el espaciotiempo es un campo que se deforma y oscila propagando estas oscilaciones en forma de ondas, llamadas ondas gravitacionales. Esto tiene una gran semejanza con la luz que es el campo electromagnético que se propaga en forma de ondas. 
En la teoría cuántica la luz se propaga en paquetes llamados fotones, los cuales tienen existencia real. En una teoría cuántica de la gravedad, la cual aún no se ha logrado desarrollar, tanto el espacio como el tiempo aparecerían en forma de corpúsculos.

La cuantización del campo gravitacional corresponde a la "granulación" del espacio-tiempo. Con esto tendríamos que el espacio y el tiempo no son continuos sino que existen en paquetes de dimensiones que tienen un valor mínimo.

En este caso tendríamos elemento de volumen y de tiempo que ya no podremos dividir más. El espacio y el tiempo se vuelven discretos. Podríamos decir que existen partículas de espacio. Lo mismo podemos decir del tiempo, que serían partículas de tiempo. En la RG cuantizada lo que tendríamos serían partículas de espacio-tiempo. A la mínima longitud espacial que se puede tener se le llama longitud de Planck y tiene un valor de $10^{-33}$ centímetros. Para el caso del tiempo el valor mínimo es de $10^{-43}$ segundos, que es llamado el Tiempo de Planck. ${ }^{1}$

\section{EL PRINCIPIO DEL TIEMPO}

En el estudio del origen del universo se requiere, por supuesto, realizar observaciones. Actualmente existen tres maneras de estudiar el pasado del universo. La primera y más antigua es mediante las ondas electromagnéticas que nos llegan del espacio. En el caso del estudio del pasado más remoto del universo, esto se realiza observando el cielo y buscando los confines del universo, es decir lo más alejado que podamos observar. Al detectar la luz o la información que nos llega de esos lugares lejanos estamos viendo cómo era el universo hace miles de millones de años. Para ver ese estado del universo en el pasado debemos dirigir los más potentes telescopios para observar las galaxias más alejadas. La luz que de ellas proviene nos da información de cómo era la fuente que emitió esta luz hace miles o millones de años.

Como fenómeno comparativo, consideramos la luz que emite nuestro Sol. Al observarla lo que vemos es como era el sol hace 8 minutos, ya que esto es lo que tarda la luz del Sol en llegar a la Tierra. Similarmente, al observar la luz de una estrella que se encuentra a 13 mil millones de años, estamos viendo como era el universo hace 13 mil millones de años.

Sin embargo, no hay manera de ver como era el universo unos minutos después del big bang debido a que no hay luz que nos lo indique. La luz más antigua en el universo es la Radiación de Fondo. Esta corresponde a una radiación de microondas, que empezó a viajar por el espacio en el momento en que el universo se volvió transparente. Esto ocurrió cuando el universo tenía una edad aproximada de 380 mil años, es decir 380 mil años después del big bang.

Mediante la observación de la luz que nos llega del espacio, no hay manera de "ver" más atrás en el pasado de nuestro universo que cuando este tenía 380 mil años de edad. Ni aun con los telescopios más potentes, puesto que no hay en el universo radiación que corresponda a una edad menor de 380 mil años. La radiación más antigua en el universo es la llamada Cosmic Background Radiation. Ya se ha detectado y nos da información de cómo era el universo a los 380 mil años del big bang.

Existe otra manera de observar el universo a una edad más temprana. Esta forma es observando un tipo de Radiación Cósmica de Fondo de Neutrinos (Cosmic Neutrino Background). Los neutrinos son partículas que interaccionan muy débilmente con la materia, y el universo se volvió transparente a los neutrinos a la edad de un segundo. Por este motivo si se llega a detectar esta radiación de fondo de neutrinos, nos traería información de cómo era el universo después de un segundo del big-bang.

Recientemente, en el año de 2017 se detectaron las ondas gravitacionales, cuya existencia teórica fue predicha por Einstein en su teoría de la Relatividad General. Este nuevo descubrimiento abre una nueva manera de observar el universo. Observar las ondas gravitacionales que se producen en los violentos fenómenos de interacción o colisión de 
estrellas de neutrones, quásares o agujeros negros nos dará una nueva información de lo que ocurre en el universo. La posibilidad de detectar las ondas gravitacionales primigenias, es decir, aquellas que se originaron en los primeros instantes del big-bang, que sería el origen del tiempo, nos podrá revelar lo que ocurrió durante los primeros instantes del universo, es decir, durante el big bang. Sin embargo, no podemos saber lo que pasó en el tiempo $t=0$, que sería el verdadero origen del tiempo. Esto representa una singularidad de la teoría general de la relatividad. Por ahora, el tiempo más corto que podemos aproximarnos al origen del universo es $10^{-43}$ segundos, que es llamado el Tiempo de Planck. Para tiempos menores que el tiempo de Planck la física que tenemos falla y deja de tener validez.

Si nos vamos hacia atrás en el tiempo hasta un instante de tiempo increíblemente pequeño después del big bang, encontramos que todo el universo ocupaba una región infinitesimal del espacio. De acuerdo con las teorías cuánticas este estado presentaría una enorme turbulencia donde se crean y se destruyen, por efectos de fluctuaciones cuánticas, enormes cantidades de materia y energía. Una violenta efervescencia de creación y destrucción de materia ocurría en esos momentos. Con esto concluimos que el comportamiento del universo en sus inicios necesariamente fue cuántico, debido a las dimensiones tan pequeñas del mismo. Por lo tanto, inicialmente el tiempo estaba cuantizado. ${ }^{2}$

\section{EL TIEMPO EN LA TEORÍA DE LA RELATIVIDAD ESPECIAL}

El tiempo en la ciencia se mide con instrumentos, con relojes o cronómetros y el hecho es que los intervalos de tiempo que miden estos instrumentos depende del marco de referencia donde se encuentren.

En la teoría de la relatividad lo primero que necesitamos definir para medir el tiempo es un marco de referencia físico. Este marco de referencia consiste en un sistema de coordenadas y un conjunto infinito de relojes colocados en cada punto del espacio. Por definición, un suceso o evento es algo que ocurre en un punto del espacio, en un instante determinado. Un evento queda determinado por un punto $(x, y, z, t)$ en un espacio de cuatro dimensiones, el espacio-tiempo.

Supondremos que un reloj es un mecanismo perfecto que mide el tiempo correctamente. Esto significa que la medición del intervalo de tiempo entre dos suceso, observados en el mismo marco de referencia, medido por dos relojes diferentes, con posiciones fijas, tiene el mismo valor para todos ellos.

De acuerdo con la teoría de la relatividad especial, al comparar los intervalos de tiempo medidos por relojes diferentes en movimiento relativo uno respecto a otro, obtendremos diferencias que dependen de la velocidad relativa entre ellos. Esencialmente, dos observadores colocados en diferentes marcos de referencia en movimiento relativo, medirán intervalos distintos de tiempo para dos sucesos.

Por otra parte, si incluimos el efecto de la gravedad, es decir, si extendemos las ideas a la Teoría General de la Relatividad (TGR), encontraremos, por ejemplo, que en la superficie de la Tierra, dos relojes colocados a diferente altura miden diferentes intervalos de tiempo, o marchan a diferentes ritmos.

En general, el intervalo de tiempo medido por dos relojes distintos depende del valor de la intensidad del campo gravitacional en el lugar donde está colocado cada reloj. Para ilustrar la idea, realicemos un experimento que ha sido ejecutado múltiples veces. Consideremos por ejemplo dos buenos relojes que se sincronizan en el marco de referencia de la Tierra, en el primer piso de un edificio de diez pisos. Si ahora llevamos uno de ellos al piso diez y lo dejamos ahí 24 horas, por ejemplo, y después lo regresamos al primer piso para comparar las lecturas de los relojes, encontraremos que hay una diferencia entre la hora que marcan ambos relojes. Observaremos que el reloj que fue llevado al décimo piso y regresado para compararlo 
con el otro, se ha adelantado. La diferencia es muy pequeña, pero con la precisión que han alcanzado los instrumentos en la actualidad, esta ha podido ser medida. La explicación de esto se encuentra en la teoría general de la relatividad de Einstein. Proviene del resultado de que en un campo gravitacional intenso los relojes marchan más lentamente que en un campo gravitacional de menor intensidad. En nuestro caso, en el décimo piso la intensidad del campo gravitacional de la Tierra es menor que en el primer piso. De aquí el resultado mencionado de que el reloj que movimos al decimo piso se adelantó.

Este hecho no es solo un resultado teórico comprobado experimentalmente, sino que es utilizado en la tecnología moderna. En los aparatos de localización que utilizamos, los GPS'es que están integrados a los teléfonos celulares y que usamos diariamente. Los satélites usados para ubicarnos aplican estos resultados de la TGR. Las señales que nos envían son corregidas de acuerdo con la altura a la que se encuentra cada satélite. Como sabemos, los automóviles autónomos que no usan conductor, usan los sistemas de información satelital. Si los carros autónomo siguiera instrucciones de satélites de ubicación GPS que no tomaran en cuenta este cambio en la medición del tiempo, los carros chocarían.

Para estudiar la teoría de la relatividad en un adecuado marco de referencia, Minkowski introdujo el concepto de un hiper-espacio de cuatro dimensiones donde la cuarta dimensión es el tiempo. En este sentido, el tiempo de la relatividad especial, es la cuarta componente de un espacio-tiempo de cuatro dimensiones. El tiempo físico medido por un reloj está dado por el tiempo propio, descrito como $d \tau=\frac{1}{c} \sqrt{d s^{2}}=\sqrt{1-\left(\frac{v}{c}\right)^{2}} d t$, donde $v$, es la velocidad del reloj respecto a un marco de referencia inercial.

En el caso de la relatividad general, $d \tau^{2}=\frac{1}{c^{2}} d s^{2}=\frac{1}{c^{2}}\left\{\left(1+\frac{2 \varphi}{c^{2}}\right) c^{2} d t^{2}+d \vec{r}^{2}\right\}$, siendo $\varphi(x, y, z)$ el valor del campo gravitacional en el punto indicado. Si el reloj está en reposo en el campo gravitacional $\varphi$ entonces $d \vec{r}=0$, y obtenemos que el tiempo $d \tau$ medido por el reloj está

dado por $d \tau=\sqrt{1+\frac{2 \varphi}{c^{2}}} d t$. Puesto que para un campo estático $\varphi=-\frac{G M}{r}$, siendo $G$ la constante de la gravitación universal, $M$ la masa que origina el campo y $r$ la distancia a la que se encuentra colocado el reloj del centro de la distribución esférica de masa. Encontramos que el reloj marcha más lentamente en presencia de un campo gravitacional que sin él. ${ }^{3}$

En general, podemos establecer que no existe un tiempo universal. En la teoría de la relatividad la presencia de materia afecta al tiempo, así como también lo afecta el movimiento relativo de los relojes.

Cuanto más nos acerquemos a un cuerpo astronómico, los relojes marcharán más lentamente. En el extremos de los cuerpos compactos, están los agujeros negros (AN). En el límite de proximidad a un $\mathrm{AN}$, en el horizonte de eventos, por la parte exterior a un agujero negro los relojes se detendrán. Si pudiéramos acercarnos en una nave espacial a un AN lo suficiente, pero por supuesto siempre fuera del horizonte de eventos, y permaneciéramos ahí durante una hora en nuestro reloj, al regresar a la Tierra, aquí habrían pasado cientos de años. En este sentido aseguramos que es posible viajar al futuro.

Similarmente dos relojes, uno fijo y el otro moviéndose respecto al primero, al comparar los intervalos de tiempo medidos por cada uno de ellos, encontramos que el que el reloj que se mueve marcha más rápidamente que el que permanece estacionario.

\section{LA FLECHA DEL TIEMPO}

Un aspecto interesante de las leyes de la física es que son invariantes ante inversiones en el tiempo. En las teorías fundamentales de la física como la mecánica clásica o la teoría 
electromagnética o la mecánica cuántica no hay distinción entre el pasado y el futuro. En el Modelo Estándar que es la teoría más acabada de la física hasta ahora, sus leyes son invariantes ante el cambio de $t \rightarrow-t$. Hasta aquí, las leyes de la física no incorporan ninguna información sobre la dirección del tiempo.

En el sistema solar, por ejemplo, no podemos distinguir el pasado del futuro. La descripción del movimiento de los cuerpos que forman el sistema solar no se modifica si cambiamos $t$ por $-t$. Esto se debe a que es un sistema de muy pocos cuerpos que se describe mediante las leyes de Newton y estas son invariantes ante la inversión del tiempo. En la microfísica no se distingue el pasado del futuro. La distinción solo ocurre en el mundo macroscópico relacionado con la entropía.

La termodinámica en su segunda ley, es la que establece la flecha del tiempo en la ciencia. Boltzmann al introducir la física estadística dejó establecido que la distinción entre presente y pasado es una propiedad estadística del comportamiento de la materia. El concepto que distingue el pasado del futuro en física es la entropía.

\section{EL TIEMPO Y LA PERCEPCIÓN HUMANA}

La percepción humana del tiempo es un acto o un proceso donde necesariamente interviene un fenómeno relacionado con un cambio. Lo ejemplificaremos con el movimiento de una partícula al considerar la posición en un instante y su posición en otro instante posterior. Si nosotros no tuviéramos memoria no percibiríamos el movimiento, solo registraríamos posiciones distintas en una escala espacial.

El cerebro humano resulta ser a la vez que un detector de posiciones, también integra e interpreta la evolución de las posiciones de la partícula creando así la sensación de movimiento y dando origen al concepto de tiempo. En este sentido el tiempo resulta ser una invención humana que no tiene existencia física. El tiempo es una experiencia humana, no algo objetivo, físico, que sea independiente del cerebro humano.

Un conjunto de estados, cada uno estático, dan la impresión de movimiento, es decir, el cerebro genera o introduce una nueva variable, el tiempo, originada en nuestra capacidad de memoria. Consideremos, por ejemplo, el fenómeno físico detrás del cine. Al proyectarse una película en una pantalla, lo que observamos es una sucesión de fotos fijas dando la impresión de movimiento. Esta sensación de movimiento se produce como consecuencia de la intervención de nuestra mente, de nuestra capacidad de memoria.

Algo semejante al fenómeno visual asociado al cine ocurre con nuestro sistema auditivo. Una pieza musical se aprecia debido a la sucesión de sonidos u ondas auditivas que son integradas en nuestra mente por nuestro cerebro debido también a la memoria.

El cerebro del ser humano no registra simplemente la información que recibe de los sentidos (la vista, el oído, etc.), sino que interpreta e introduce elementos adicionales a la sola señal luminosa o auditiva. Esto es esencialmente lo que ocurre al construir en la mente la percepción del tiempo.

¿Qué es lo que vemos, longitudes de onda o imágenes?, la misma pregunta para los sonidos, ¿Qué es lo que oímos, longitudes de onda o sonidos? Supongamos que escuchamos las voces de varias personas a la vez. Nosotros tenemos la capacidad de seleccionar cualquiera de ellas y escuchar lo que dice solo una persona, aun cuando todas las vibraciones lleguen a nuestro oído al mismo tiempo. Con este ejemplo, encontramos que nuestro oído combinado con el funcionamiento del cerebro da como resultado la percepción auditiva. Lo mismo ocurre con la percepción visual.

Concluimos que la percepción del tiempo, como la auditiva y visual, está integrada por varios elementos psíquicos en los que interviene la memoria humana. ¿Qué pasaría si no tubieramos 
memoria? Esta pregunta se antoja hacerla debido que queremos construir teorías físicas objetivas, independientes de la conciencia humana o de factores psicológicos. Esta línea de razonamiento, nos lleva al propósito de lograr construir una teoría física objetiva, sin que participen factores subjetivos. Por lo tanto, esta teoría física no debería contener la variable tiempo. ${ }^{4}$

Los fenómenos de ilusión óptica nos indican que nuestros sentidos pueden engañarnos. Lo que se busca al elaborar una teoría física es que esté a salvo de los efectos psicológicos, es decir evitar la influencia de lo que nuestro cerebro interpreta y que da lugar a las ilusiones ópticas y auditivas.

\section{LA TEORÍA CUÁNTICA DE LA GRAVEDAD Y LA UNIFICACIÓN DE TODA LA FÍSICA}

La física pretende ser totalmente objetiva, que ningún elemento psicológico, como preferencias o interpretaciones esté presente en las teorías físicas. El concepto tiempo, por lo que hemos mencionado, tiene importantes elementos psicológicos. Por esta razón se busca construir una teoría física sin tiempo. La percepción humana, la cual tiene fuertes elementos subjetivos, no debe aparecer en la estructura de una teoría física. La percepción humana, como ya lo dijimos, incluye en la observación muchos ingredientes psicológicos, que una teoría física debe eliminar. Una teoría física debe contener solamente lo que se registra en un aparato, y no deben entrar en ella elementos o factores de naturaleza psicológica. Hay algunos científicos que piensan que no se ha logrado construir la Teoría Cuántica de la Gravedad (TCG), debido a que no se ha eliminado el tiempo en las formulaciones que se han intentado. La opinión de algunos científicos, líderes en la búsqueda de una TCG, establecen que esta se logrará desarrollar cuando se tenga un formalismo matemático donde el tiempo quede excluido. ${ }^{5}$

\section{LOS VIAJES EN EL TIEMPO}

El tema de los viajes en el tiempo ha sido tradicionalmente considerado como un asunto de la ciencia de ficción o la fantasía. Sin embargo, la física moderna se ocupa hoy de este tema como una cuestión estrictamente científica.

A semejanza de las fantasías de la literatura sobre alfombras mágicas o bolas de cristal, que hoy vemos materializadas en los aviones y en la televisión, es probable que los viajes en el tiempo sean también una realidad dentro de quinientos o mil años.

De acuerdo con lo que hoy sabemos de la física, es posible que la tecnología del futuro haga realidad los viajes en el tiempo. Si nos adentramos en los fundamentos de la física que hoy conocemos, los viajes en el tiempo no son imposibles.

Sin pretender asegurar que en el futuro se lograrán estos viajes en el tiempo, la idea es establecer que la física no los prohíbe. Un principio de la física asegura que lo que no está prohibido por las leyes de la naturaleza, seguramente ocurrirá. Ese es el criterio aplicado para cualquier fenómeno o proceso que no se ha observado pero que no está prohibido por las leyes de la naturaleza. En el caso de los viajes en el tiempo, si este es el caso, la limitación es actualmente de tipo tecnológico.

La historia de la ciencia está llena de anécdotas sobre opiniones negativas y pesimistas de connotados científicos sobre el futuro de la ciencia y la tecnología. Esto debe normar nuestra forma de pensar antes de descartar un desarrollo científico o tecnológico. Los alquimistas pensaban en descubrir la Piedra Filosofal, algo que sería capaz de convertir los metales sin valor como el plomo, en oro. Un sueño que se consideró imposible. Actualmente esto es una realidad en los laboratorios de física nuclear donde la conversión o transmutación de unos núcleos atómicos en otros, se realiza mediante el bombardeo con partículas. 
Stephen Hawking intentó probar que los viajes en el tiempo son imposibles. Buscaba encontrar una ley de la física que impidiera estos viajes. Trataba de demostrar que existe una línea cronológica en los acontecimientos o eventos, como se les llama en la física, que no puede ser modificada. Su intento no tuvo éxito y terminó él mismo aceptando la posibilidad de los viajes en el tiempo. Su idea de viajes al pasado la sustentó en la teoría de cuerdas llamada teoría M. De acuerdo con el conocimiento científico presente, no podemos excluir la posibilidad de los viajes en el tiempo, escribió en su libro póstumo "Brief Answers to the Big Questions". http://xn-webducation-dbb.com/wp-content/uploads/2019/01/Stephen-Hawking-Brief-Answers-to-the-

Big-Questions-Random-House-Publishing-Group-2018.pdf. ${ }^{6}$

La conclusión, basada en los principios de la física, en concordancia con el principio de legalidad que establece que todo lo que no está prohibido, está permitido, es que si algún fenómeno o proceso no está prohibida por las leyes de la naturaleza, este fenómeno ocurrirá, tarde o temprano. Puede ser dentro de cien años, o mil o un millón de años, pero sucederá.

\section{VIAJES AL FUTURO}

El viaje al futuro es un asunto cotidiano de acuerdo con la teoría de la relatividad. Al movernos en una nave o un avión, estamos viajando hacia el futuro de acuerdo con nuestro reloj biológico, ya que los relojes marchan más lentamente que los relojes en reposo. Al comparar los dos tiempos en los marcos de referencia respectivos, encontramos que el reloj que se mueva viaja hacia el futuro ya que encuentra cosas en el futuro para su tiempo propio. La vida de un individuo que viaja se prolonga respecto a su vida en reposo. En este sentido se adelanta a su propio tiempo, es decir, viaja al futuro. Un futuro que no hubiera podido ver o vivir si no hubiera estado en movimiento.

El problema de viajar al futuro es que ya no se puede regresar al tiempo regular, digámoslo así. Un viajero espacial que viaje durante un año, puede regresara a la Tierra donde han transcurrido, cinco o diez o más años. Una vez en la Tierra, ya no puede regresar a su tiempo, a su generación, es decir, al pasado.

La Teoría Especial de la Relatividad (TER) ha establecido firmemente que se puede viajar al futuro, lo cual ha sido comprobado en múltiples experimentos. Como ya se mencionó, un reloj en movimiento marcha más lentamente que uno estacionario. Esto se comprueba en experimentos con partículas inestables. Una partícula inestable tiene un tiempo de vida promedio de un cierto valor. La partícula se crea en un instante determinado y después de su tiempo de vida media, en términos estadísticos, se desintegra. Podemos calcular, mediante una estimación sencilla qué distancia puede recorrer esta partícula antes de desintegrarse. Un ejemplo de esto son las partículas llamadas muones, que se producen en las capas superiores de la atmósfera a mucho mas de 6,000 metros de la superficie del mar. La vida media de estas partículas es de $2 \times 10^{-6}$ segundos. En este tiempo, moviéndose a la velocidad de la luz, recorrerían cuando mucho 600 metros. Sin embargo, se les detecta al nivel del mar, a seis mil metros de donde se crearon. La explicación de esto es el fenómeno conocido en la relatividad como dilataciones tiempo. Esto significa que aun cuando en el "reloj" del muon hayan transcurrido menos de $2 \times 10^{-6}$ segundos, para un observador en la Tierra transcurrió un tiempo diez veces mayor.

Pensemos que el muon tiene un reloj integrado a sí mismo. El resultado anterior es equivalente a que antes de morir pudo vivir diez veces más tiempo que el que le corresponde a su vida media. Extendamos la idea a una persona que viaja desde algún punto del espacio hacia la tierra durante un año en su propio reloj, marcado por los latidos de su corazón. Este año es lo que ha envejecido el viajero. Dependiendo de la velocidad con la que esté viajando, puede ocurrir que 
aquí en la Tierra hayan transcurrido, por ejemplo, veinte años o cincuenta, por ejemplo. En este sentido el viajero viajó al futuro, cuarenta y nueva años o 99, según el caso.

Los relojes atómicos son los más precisos de entre todos los relojes. Con estos instrumentos somos capaces de medir la variación en tiempo entre el tiempo medido por un reloj de pulso usado por un astronauta en un viaje y el tiempo registrado por un reloj aquí en la Tierra.

Por otra parte, la TGR establece que el tiempo no es universal y que cada planeta o estrella dependiendo de su campo gravitacional tiene su propio tiempo.

En síntesis, los viajes al futuro, no presentan ninguna dificultad conceptual que contravenga ni la lógica ni lo que hoy sabemos científicamente.

\section{POSIBILIDAD DE VIAJAR AL PASADO}

Las dos teorías fundamentales de la física, son la Teoría General de la Relatividad (TGR) y la Mecánica Cuántica (MC). La TGR admite soluciones de las ecuaciones de Einstein que son cíclicas en el tiempo, lo que implica que la teoría no prohibe que se puede viajar en el tiempo. Sin embargo, existen contradicciones lógicas que deben superarse si se admite científicamente que esta posibilidad sea real.

En una histórica conferencia dictada en 1949 por el distinguido matemático Kurt Goedel, en el Instituto de Estudios Avanzados de Princeton, EUA, presentó una prueba matemáticamente, basado en la teoría de Einstein de la relatividad general, de que el tiempo es cíclico y que lo que en este momento estamos viviendo, se repetirá exactamente igual, con los mismos protagonistas, usted leyendo este texto, en el mismo lugar en el que ahora lo hace y rodeado del mismo entorno, dentro de miles de millones de años. Algo que parece escalofriante. ${ }^{7}$

Impactado por el resultado obtenido por Goedel, Einstein recurrió a la filosofía para descartar el resultado. Su argumento fue que ante resultados matemáticos que choquen con la experiencia, se debe recurrir a la intuición. Einstein, que creía en la realidad última del espacio-tiempo recurría ahora a la intuición para desechar el resultado, no físico según él, obtenido por Goedel.

Motivado por este resultado, Goedel buscó establecer cuáles son los límites de la matemática. Cuando su uso conduce a resultados que contradicen conceptos intuitivos. Esto nos lleva de nuevo a uno de los problemas filosóficos fundamentales de la ciencia. A quién creer o en qué basarnos para construir el conocimiento, una estructura teórica confiable que describa la realidad física. Esta situación presenta la disyuntiva filosófica del racionalismo contra el empirismo.

Estas soluciones de las ecuaciones de Einstein que permiten viajar al pasado, son llamadas Curvas Temporales Cerradas o Closed Timelike Curves (CTC), son físicamente aceptables. El problema que se tiene ahora, es el de resolver los problemas lógicos que se presentan al viajar al pasado, como la paradoja de abuelo, de la que hablaremos enseguida.

\section{LA PARADOJA DEL ABUELO}

El primer punto escabroso sobre los viajes al pasado es el hecho fundamental de que el pasado no puede ser modificado y alguien que viaje al pasado puede alterar alguna cosa que haría que el presente no fuera posible como lo vemos actualmente. Este es el origen de la muy conocida Paradoja del Abuelo. La esencia de esta paradoja es que un viajero que va hacia el pasado se encentre con uno de sus ascendientes, es decir, con una persona de su árbol genealógico y lo mate. Esta posibilidad provocaría una contradicción entre el presente y el pasado. Descender de alguien que murió antes de tener hijos y por lo tanto ser hijo de alguien que nunca existió es una contradicción lógicamente inaceptable.

Cualquier cosa que haga una persona que viaje al pasado puede inhibir algo que está conectado causalmente con el presente y por lo tanto muchas cosas del presente deberían desaparecer 
instantáneamente. La misma posibilidad de que el viajero asesine a su abuelo destruye el argumento de que viaje alguien que no existiría. Esta contradicción lógica se ha usado para invalidar la posibilidad de viajar en el tiempo hacia el pasado.

Los intentos actuales para explicar y resolver los problemas de los viajes al pasado, se centran en encontrar una salida a la paradoja del abuelo. El camino que se ha seguido es el de buscar en la mecánica cuántica una justificación y poder hacer a un lado la paradoja del abuelo. Puesto que la mecánica cuántica es una teoría no causal, es decir, no tiene validez el principio de causalidad, es posible encontrar una salida al problema de la paradoja. ${ }^{8}$

\section{LA MECÁNICA CUÁNTICA}

Es importante establecer que en la mecánica cuántica la intuición no es un recurso mental de confianza. Los extraños y misteriosos aspectos de la mecánica cuántica hacen de ella un asignatura difícil de entender. Las paradojas que se presentan en el planteamiento de los viajes en el tiempo, como la paradoja del abuelo, son resueltas por la mecánica cuántica. Esto se explica al considerar los postulados de la mecánica cuántica, donde se establece que en una medición se produce el efecto de colapso de la función de onda en el estado que arroja la medición. La realidad no es independiente del observador. El observador perturba al sistema.

Existen dos tipos de soluciones a las contradicciones que se presentan al viajar al pasado. Uno de los tipos se clasifica en imponer restricciones a lo que es posible que el viajero en el tiempo puede hacer. Todo aquello que entre en contradicción lógica con el futuro no lo puede hacer el viajero en el tiempo.

El otro tipo de solución a las contradicciones lógicas es que cuando el viajero regrese aparecerá en un universo paralelo. Esto es, regresa a un futuro alternativo. Esta posibilidad está aceptada por la mecánica cuántica. En estos casos el viajero puede interactuar consigo mismo en el pasado pero todo debe ser consistente con el futuro. Por ejemplo, el viajero no puede matar a un antepasado suyo. La explicación a esto es que si el viajero pretende hacerlo, entonces en el momento de intentar matar a su abuelo, por ejemplo, ocurrirá una fluctuación cuántica que impide hacerlo, haciendo que falle, por ejemplo la pistola con la que lo iba a matar.

Existe un tipo de paradoja relacionada con problemas matemáticos. Esta es conocida como la paradoja del teorema no probado. Por ejemplo, un teorema se prueba y se escribe en un libro que una persona lee. Esta persona viaja al pasado y le muestra la demostración a un matemático. El matemático incluye esta demostración en un libro de su autoría, y este libro resulta ser el mismo en el que el viajero lo leyó. La paradoja reside en que el viajero obtuvo la demostración del teorema de algo que no existía. Pero en esencia esta es la misma paradoja que la del abuelo, ya que al encontrar él a su abuelo el viajero nació de alguien, su padre, que todavía no existía. Lo mismo la demostración del teorema se obtuvo de algo que no existía. Ambas situaciones resultan ser esencialmente el mismo problema.

Todos los tipos de paradojas que se han encontrado, son esencialmente del mismo tipo de la del abuelo. La mecánica cuántica resuelve todos estos tipos de paradojas. Sea suponiendo la existencia de muchos universos (universos paralelos), también llamada versión de muchos mundos, o la hipótesis de NOVIRKOV. ${ }^{9}$

Lo importante de la incorporación de la mecánica cuántica a una teoría general de la relatividad es que se resuelven las paradojas que se presentan en los viajes al pasado, como la paradoja del abuelo. Al permitir la TGR las soluciones CTC y además se incorporan en la TGR las ideas de la mecánica cuántica, el asunto de los viajes al pasado resultan en una posibilidad real. ${ }^{10}$ 


\section{PRINCIPIO DE CAUSALIDAD}

Por otra parte, el principio de causalidad establece que una causa precede siempre a un efecto. Si este principio, que no es una ley de la naturaleza o que se derive de las leyes fundamentales de la física, es verdadero, entonces los viajes en el tiempo adolecen de una fuerte contradicción lógica. La paradoja llamada del abuelo, anularía la posibilidad de los viajes en el tiempo. Esta paradoja está basada en la validez del principio de causalidad, donde la línea de tiempo debe respetarse rigurosamente. Es decir, primero nace el abuelo, luego su padre y después el viajero. Si el abuelo no existió entonces no puede existir el viajero.

La salida al problema de la paradoja del abuelo está en que la mecánica cuántica es una teoría no causal. Por lo tanto la línea de tiempo no se respeta en el comportamiento cuántico, Este hecho es el que ha abierto la posibilidad del estudio científico de los viajes en el tiempo. Si en la mecánica cuántica no se cumple el principio de causalidad, entonces los viajes al pasado son posibles.

Los viajes en el tiempo han sido tratados en los libros de ciencia de ficción y en películas de fantasía y ciencia ficción. Lo que se concluye de este artículo que aborda la parte científica del tema, es que, como se dice usualmente, los viajes en el tiempo son un asunto te tiempo.

\section{PRINCIPIO DE NOVIKOV}

El Principio de Novikov establece que no puede ocurrir que al viajar al pasado el viajero realice una acción que implique una violación o alteración del futuro que se está viviendo. Este principio implica que solo hay una línea de tiempo y no podemos alterarla al viajar al pasado. Si ocurriera que se violara el principio de Novikov y pudiéramos alterar la línea de tiempo, la consecuencia, según la mecánica cuántica, es que caeríamos en un universo paralelo. Esto significa que el viajero viviría ahora en un universo distinto y desconectado causalmente del universo de donde partió. ${ }^{11}$

Es así como el Principio de Novikov, establece que el futuro es inmutable si ya ocurrió. Existe una conjetura planteada por Hawking, conocida como Chronology Protection Conjecture, la cual establece que el principio de causalidad siempre será preservado. Si no ocurre así, el universo resulta ser inestable y se colapsaría en forma catastrófica. Cada momento es único y no se repetirá jamás. ${ }^{12}$

Las leyes de la mecánica cuántica resuelven el problema de la paradoja del abuelo y, en principio, permiten los viajes al pasado. El concepto de múltiples líneas de tiempo donde un suceso ocurre de dos o más formas distintas determina las formas diferentes en las que evolucionaron los acontecimientos.

$\mathrm{Si}$ aceptamos una teoría que respete el principio de causalidad, los viajes al pasado quedan prohibidos por la misma teoría. Sin embargo, considerando que la teoría cuántica es una teoría no causal, se abre la posibilidad de los viaje en el tiempo.

En general, si las leyes que gobiernan los procesos de la naturaleza están regidas por el principio de causalidad, entonces los viajes al pasado no son posibles. La mecánica cuántica no respeta el principio de causalidad, por lo tanto, las leyes de la mecánica cuántica no prohíben los viajes al pasado.

\section{MAQUINAS DE TIEMPO GRAVITACIONALES}

Hawking, quien no creía en la posibilidad de los viajes en el tiempo, se convenció de que, de acuerdo con las teorías físicas actuales, estas no prohibían los viajes en el tiempo y que las soluciones CTC (Closed Timelike Curves) son físicamente aceptables. Una curva temporal cerrada es una manera de decir, en el lenguaje de la teoría general de la relatividad, que se puede viajar en el tiempo, particularmente al pasado. Una curva temporal cerrada es una solución 
matemática, físicamente aceptable de las ecuaciones de campo de la relatividad general, en la cual, la línea mundo o la línea que sigue un objeto (una persona) en el espacio-tiempo, sigue una trayectoria que lo lleva al mimo punto en el pasado. En consecuencia, el objeto (la persona) viaja al pasado. ${ }^{13,14}$

Para evitar el problema de la paradoja del abuelo, sin considerar las teorías cuánticas, Hawking introdujo una conjetura llamada Chronology Protection Conjecture. Esta conjetura la estableció Hawking para prevenir que se cambie el pasado. Algo debe evitar que alguien viaje al pasado y produzca un cambio que altere la línea cronológica. Esta suposición de Hawking lleva implícita la idea de que el viajero es parte del pasado y no puede alterarlo. Al ser la línea mundo del viajero una línea cerrada, esta lo llevará al mismo punto del espacio-tiempo en el futuro. Por lo tanto no producirá ninguna alteración. Esta fue la idea de Hawking al imponer su conjetura de la protección cronológica.

La física de los agujeros de gusano establece que los agujeros de gusano son el equivalente a máquinas para viajar en el tiempo. ${ }^{15}$ La posibilidad de construir una máquina del tiempo de agujeros de gusano sin ser despedazados depende de las leyes que gobiernen la teoría cautica de la gravedad, las cuales todavía no conocemos. ${ }^{16}$

Cualquier cosa que no esté prohibida por las leyes de la naturaleza será lograda con las tecnologías del futuro, por más imposible que parezca. No olvidemos que la civilización puede durar miles de años más.

\section{EL FUTURO DEL SER HUMANO}

Muy probablemente dentro de doscientos años, el ser humano como lo conocemos ahora desaparecerá. Serán seres formados por otros materiales, con un cerebro, que es donde radica la esencia del ser humano, que tendrá componentes materiales inorgánicos donde se almacenará información y conocimientos. El ser biológico que somos en la actualidad se habrá transformado. Estará repleto de prótesis que mejoraran nuestros sentidos actuales, visión, audición, olfato, gusto y tacto y nuestra memoria también será mejorada.

Un ser humano inorgánico reemplazará al ser humano biológico, orgánico. Con el desarrollo de las tecnologías de la información y de la inteligencia artificial, el hombre del futuro, transformado por la ingeniería genética, o la ingeniería biológica, será totalmente diferente a nosotros.

El hombre del futuro cambiará su propio ADN con lo cual transformará sus capacidades mentales y físicas. Los grandes problemas del ser humano del futuro serán de naturaleza ética y moral, es decir, cómo legalizar o legislar lo que se puede hacer y lo que no se permite, con un ser humano desde su embrión. Las leyes que hoy nos gobiernan quedarán muy por detrás de los avances tecnológicos y las posibilidades de hacer cambios en nuestro cuerpo, nuestra mente y nuestro ADN quedarán limitados por las leyes que nosotros mismos impongamos.

Modificando el ADN de cualquier animal, podemos crear uno muevo, mejorado, en el sentido de que sea más inteligente o mas fuerte o más resistente a las enfermedades y las plagas. Esto ya se hace con plantas, las famosas plantas transgénicas. El mundo del futuro se puede ya vislumbrar para dentro de 50 años, pero el de aquí a cien años, aun con la que ya tenemos de tecnología genética y biología molecular, no podemos siquiera imaginarlo.

Podremos tener nano-robots que se inyectan al cuerpo y circular por el sistema sanguíneo y detecten enfermedades o virus que ocasionan enfermedades y destruirlos. Esto sería una especie de sistema inmunológico externo, agregado a nuestro sistema inmunológico natural.

Con toda esta nueva tecnología del futuro, ¿Serán posibles en el futuro los viajes en el tiempo, al pasado? Si el problema es de tipo tecnológico, desde ahora podemos afirmar que sí serán posibles. Esta afirmación se hace basada en que la tecnología seguirá avanzando y lo que hoy 
nos parece imposible, como ha ocurrido en la historia de nuestra civilización, en el futuro será logrado, materializándose en hechos concretos. La limitación queda impuesta por las leyes de la naturaleza, no de la tecnología. Pensemos en la tecnología que habrá desarrollado el ser humano en los siguientes cinco mil años, por ejemplo. ¿Pensaremos que no lograremos algo que la naturaleza no lo prohíba? Históricamente se ha probado que lo que parecía imposible se ha vuelto realidad.

La tecnología que se requiere para realizar viajes en el tiempo está muy lejos de ser alcanzada, pero posiblemente dentro de mil años o más se logrará desarrollar. ${ }^{17}$

\section{EPÍLOGO}

Quienes trabajan en la teoría cuántica de la gravedad pretenden construir una ecuación cuántica relativista que no contenga el tiempo como variable. La pretensión de eliminar el tiempo es debido a que este concepto no tiene un significado físico importante. Solo cuando se incluye la entropía o el calor el concepto tiempo es realmente fundamental. Parece ser que el camino para construir una teoría cuántica de la gravedad es eliminar la variable tiempo de la formulación matemática.

La idea de que el tiempo pasa o fluye lo hace diferente al espacio, el cual no tiene intuitivamente el carácter de algo que fluye o pasa. Un lugar físico en el espacio está ahí y no cambia su localización, mientras que un instante de tiempo pasa y se pierde para siempre. ¿Es esto correcto? ¿Existe un reloj cósmico universal que marque el tiempo igual para todos? No, no existe. El tiempo es relativo. ¿El pasado ya no existe en el mundo?, es decir ¿ha desaparecido el pasado? No lo sabemos.

Los avances en el conocimiento científico que ya tenemos actualmente son tan impresionantes que la tecnología que con ellos se puede desarrollar no la podemos siquiera imaginar ahora. Aun hoy que estamos padeciendo la pandemia del Covid 19, podemos decir que es muy probable que dentro de cien años hayan desaparecido la mayoría de las enfermedades que hoy tenemos, como las enfermedades cardiacas, degenerativas, mentales, y muchas otras. El desarrollo de la inteligencia artificial, la robótica, la medicina, la biología y la física nos permitirá prolongar la vida de los seres humanos mucho más allá de los cien años.

Hay cosas muy interesantes, basadas en la ciencia, que se están haciendo en la actualidad para intentar esta aventura de viajar en el tiempo. Una cosa valiosa se debe sacar de esto: debido a lo apasionante del tema, muchos jóvenes se interesarían por estudiar ciencias y ya con esto el mundo sería mejor.

\section{BIBLIOGRAFÍA}

1. Smolin, Lee, (2001), Three Roads to Quantum Gravity, Basic Books, USA.

2. Rovelli, Carlo,( 2017), Reality is not what it Seems, Riverhead Books, USA.

3. Schutz, Bernard, (2002), A First Course in Ganaral Relativity, Cambridge university Press, UK.

4. Yourgrau, Palle, (2005), A World Without Time, Basic Books, USA.

5. Rovelli, Carlo, (2007), Quantum Gravity, Cambridge University Press, UK.

6. Hawking, Stephen (2018), Brief Answers to the Big Questions http://xn-webducation-dbb.com/wp-content/uploads/2019/01/Stephen-Hawking-Brief-

Answers-to-the-Big-Questions-Random-House-Publishing-Group-2018.pdf.

7. (Rindler, Wolfgang, 2009). American Journal of Physics 77, 498 (2009), DOI: https://doi.org/10.1119/1.3086933

8. Bruce, Colin, (2004), Schroedinger's Rabbits, Joseph Henry Press, USA. 
9. Costa F. and Shrapnel S. (2016), Quantum Causal Modelling, New J. Phys. 18 063032.

10. Pegg, D. T., (2001). Quantum mechanics and the time travel paradox Time's Arrows, Quantum Measurement and Superluminal Behavior ed D Mugnai, A Ranfagni and L S Schulman (Roma: Consiglio Nazionale Delle Richerche) p 113.

11. Novikov, I. D., (1989), An analysis of the operation of a time machine Soviet Phys. JEPT 68 439-43.

12. Hawking. S. W., (1992). Chronology protection conjecture Phys. Rev. D 46 603-11.

13. Alcubierre, M., (1994), The warp drive: hyper-fast travel within general relativity Class. Quantum Grav. 11 L73-7.

14. Tobar, Germain and Costa, Fabio, Classical and Quantum Gravity, https://iopscience.iop.org/article/10.1088/1361-6382/aba4bc/pdf.

15. Visser, Matt, Nuclear Physics B, April, 1994, Pages 895-906.

16. Morris, M. S. and Thorne, K. S. Scientific American, (2002).

17. Michio Kaku, (2008),Physics of the Impossible, Anchor Books, USA. 